\title{
Hepatomegalia como forma de presentación en pericarditis constrictiva. Caso clínico pediátrico
}

Hepatomegaly as a form of presentation in constrictive pericarditis. A pediatric clinical case

\author{
Dra. María del R. Ortolá Martíneza, Dra. Julia Dvorkina, Dr. Gustavo Sollitto ${ }^{a}$, Dr. Willy Conejeros ${ }^{b}$ \\ Dr. Manuel Garrido y Dra. Mariana Cazalas ${ }^{c}$
}

\section{RESUMEN}

La pericarditis constrictiva es una entidad poco frecuente en pediatría, en la cual existe una limitación para la diástole cardíaca por fibrosis del pericardio. Elorigen etiopatogénico de esta patología es múltiple, encontrándose en primer lugar, la pericarditis constrictiva idiopática y, en segundo, la infección por Mycobacterium tuberculosis. El diagnóstico constituye un desafío clínico, ya que requiere de un alto grado de sospecha. Suele presentarse de forma oligosintomática. La presencia de edema, ascitis y alteración de la función hepática suele orientar el estudio hacia una enfermedad hepática primaria. Una cuidadosa historia clínica y examen físico, junto con estudios por imágenes adecuados, constituyen las piedras angulares del diagnóstico. El tratamiento quirúrgico realizado de forma oportuna resulta curativo en la gran mayoría de los pacientes. Se presenta el caso de un paciente de 16 años que inicia estudios por hallazgo de hepatomegalia asociada a disnea grado 1-2 en un control de salud habitual.

Palabras clave: hepatomegalia, pericarditis constrictiva, niño.

\begin{abstract}
Constrictive pericarditis is a rare entity in pediatrics in which there is a limitation for cardiac diastole due to fibrosis of the pericardium. The etiopathogenic origin of this pathology is multiple, finding idiopathic constrictive pericarditis firstly and Mycobacterium tuberculosis infection secondly. Diagnosis is a clinical challenge since it requires a high degree of suspicion. It usually presents as oligosymptomatic or with signs and symptoms of low cardiac output. The presence of edema, ascites and impaired liver function usually guides the study towards primary liver disease. A careful clinical history and physical examination together with adequate imaging studies are the cornerstones of the diagnosis. Surgical treatment is curative in the vast majority of patients. We present the case of a 16-yearold patient with hepatomegaly and dyspnea grade 1-2 found in a routine health check-up.

Key words: hepatomegaly, constrictive pericarditis, child.
\end{abstract}

a. Unidad 4 de Clínica Médica.

b. Servicio de Cirugía Cardiovascular.

c. Servicio de Cardiología.

Hospital de Niños Ricardo Gutiérrez,

Ciudad Autónoma de Buenos Aires.

Correspondencia:

Dra. María del R. Ortolá Martínez:

r.ortolamartinez@gmail.com

Financiamiento: Ninguno.

Conflicto de intereses: Ninguno que declarar.

Recibido: 6-11-2018

Aceptado: 23-4-2019 http: / / dx.doi.org/10.5546/ aap.2019.e523

Cómo citar: Ortolá Martínez MR, Dvorkin J, Sollitto G, Conejeros $\mathrm{W}$, et al. Hepatomegalia como forma de presentación en pericarditis constrictiva. Caso clínico pediátrico. Arch Argent Pediatr 2019; 117(5):e523-e526.

\section{INTRODUCCIÓN}

La pericarditis constrictiva es una entidad infrecuente en pediatría, en la cual el corazón se encuentra restringido por un pericardio engrosado y rígido que impide la correcta contracción miocárdica. Puede constituir el estadio evolutivo final de procesos inflamatorios que afectan al pericardio, aunque, en un alto porcentaje de casos, no puede identificarse un origen etiológico concreto. ${ }^{1}$ La principal causa infecciosa en nuestra población es la tuberculosis. ${ }^{2}$ El mayor desafío que presenta es el de realizar un diagnóstico oportuno, ya que suele presentarse de forma oligosintomática y pasar desapercibida hasta llegar a fases avanzadas de la enfermedad. ${ }^{3}$ Es por ello que es importante conocer esta patología y el rendimiento de las distintas pruebas complementarias que aportan información diagnóstica, dado que el tratamiento definitivo instaurado de forma precoz es esencial para un pronóstico favorable.

\section{CASO CLÍNICO}

Paciente de 16 años, previamente sano, oriundo de Misiones, con vacunación completa para la edad, en quien se constató, en un control de salud, hepatomegalia sin otra signosintomatología asociada. En el interrogatorio, surgió únicamente la presencia de disnea grado 1-2 ocasional y el aumento de peso en los últimos 6 meses sin cambios en los hábitos alimentarios. Se solicitó, en primera instancia, un hepatograma, en el que se evidenciaron valores aumentados de transaminasas y bilirrubina de predominio indirecto (transaminasa glutámicooxalacética: $80 \mathrm{mg} / \mathrm{dl}$; transaminasa glutámicopirúvica: $68 \mathrm{mg} / \mathrm{dl}$; bilirrubina total: $2,17 \mathrm{mg} / \mathrm{dl}$; 
bilirrubina indirecta: $1,90 \mathrm{mg} / \mathrm{dl}$ ), y una ecografía abdominotorácica, que informó hepatomegalia, líquido libre en la cavidad abdominal y derrame pleural bilateral leve. Continuó los estudios de forma ambulatoria sin lograr determinar la etiología, por lo que se decidió derivarlo para internación, estudio y tratamiento a un hospital de tercer nivel de atención.

Ingresó en buen estado general, afebril, normohidratado y normocoloreado, con peso de 64,500 kg (P75-90) y talla e índice de masa corporal dentro de los parámetros normales. Presentaba distensión abdominal leve, con matidez ante la percusión en los flancos, las fosas ilíacas y el hipogastrio, compatible con ascitis. Refería dolor leve en el hipocondrio derecho ante la compresión y se evidenciaba, por palpación y percusión, el hígado aumentado de tamaño y consistencia (medidas: 7-11-12; altura total: 12 $\mathrm{cm})$.

Se solicitó un nuevo hepatograma, en el que se evidenció la persistencia de niveles altos de bilirrubina total $(2,27 \mathrm{mg} / \mathrm{dl})$ de predominio indirecto $(1,51 \mathrm{mg} / \mathrm{dl})$. Se solicitaron serologías para virus hepatotropos, negativos, y dosaje de alfa-1-antitripsina, alfa-fetoproteína, subunidad beta de la gonadotropina coriónica humana (beta subunit of human chorionic gonadotropin; BHCG, por sus siglas en inglés) y ceruloplasmina para descartar patología oncológica hepática. Todas estuvieron dentro de los límites normales. Se realizó cupruria, que resultó negativa, y una prueba de sensibilidad a la tuberculina de $0 \mathrm{~mm}$. Se solicitó también un electrocardiograma, en el que no se evidenciaron alteraciones eléctricas de la conducción, y un ecocardiograma doppler color, que demostró dilatación biauricular, prolapso de la válvula mitral, insuficiencia mitral de leve a moderada, septum interventricular disquinético, función sistólica del ventrículo izquierdo conservada, sin engrosamiento ni aumento de la refringencia pericárdica.

Dada la presencia de síndrome ascítico, inició el tratamiento con espironolactona y se constató la disminución progresiva de la hepatomegalia y el descenso de $8 \mathrm{~kg}$ de peso con mejoría simultánea de la disnea. Se interconsultó con el Servicio de Cardiología, quienes solicitaron una angiotomografía de tórax y abdomen con contraste, en la que se halló dilatación de la vena cava inferior (VCI) suprahepática y engrosamiento pericárdico. Debido a la sospecha de patología cardiovascular con hipertensión pulmonar, el paciente fue sometido a un cateterismo cardíaco, en el que se constató presión pulmonar media aumentada $(33 \mathrm{mmHg}$ ).

Una vez descartada la cardiopatía estructural y con el hallazgo de hipertensión pulmonar, se solicitó, para hacer el diagnóstico diferencial entre patología pericárdica y miocárdica, una resonancia magnética cardíaca (Figura 1). En esta, se observó el pericardio engrosado (mayor de 4 $\mathrm{mm}$ ) con signos de fibrosis difusa y calcificaciones, hipointenso en T1 y T2 (datos compatibles con pericarditis constrictiva). Por la persistencia de los síntomas a pesar del tratamiento médico, se decidió realizar una intervención quirúrgica. Durante la cirugía, se observó el pericardio gravemente engrosado, rígido y muy adherido al corazón, y aquinesia miocárdica (Figura 2). Se realizó una pericardiectomía con resección de pericardio parietal y visceral de la cara anterior, lateral y posterior del corazón. Una vez resecado el pericardio, se observó muy buena movilidad cardíaca con buena contracción.

Dado que la tuberculosis es, en nuestra población, la primera causa infecciosa asociada a la pericarditis constrictiva, se realizó una interconsulta con el Servicio de Tisiología, quienes indicaron iniciar el tratamiento con drogas tuberculostáticas (isoniazida: $300 \mathrm{mg}$; rifampicina: $600 \mathrm{mg}$; pirazinamida: $2000 \mathrm{mg}$; y etambutol: $1800 \mathrm{mg}$ ). Se recibió luego el resultado del cultivo y de la anatomía patológica del

\section{FIgURA 1. Resonancia magnética cardíaca}

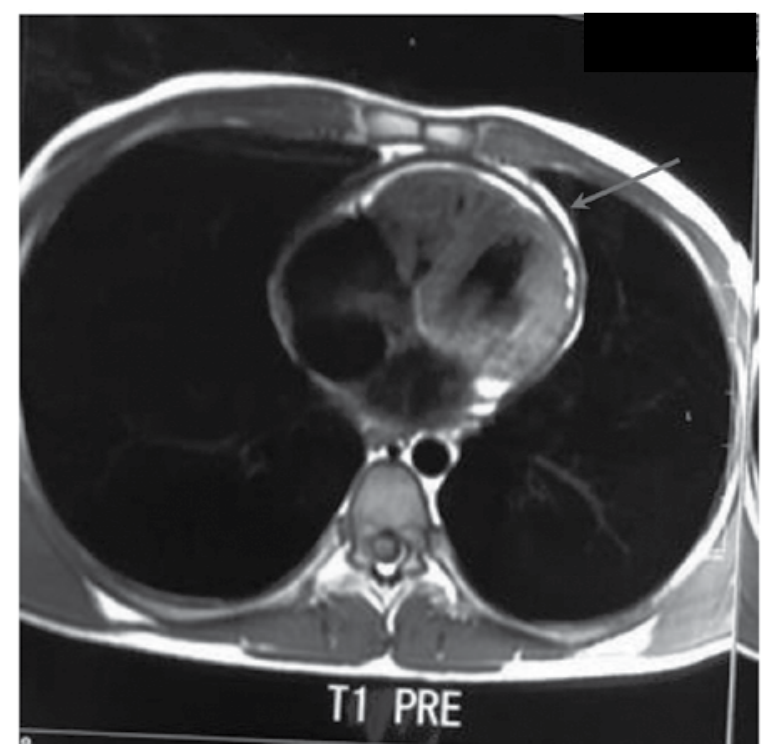

En la resonancia magnética cardíaca, se observa pericardio engrosado, compatible con pericarditis constrictiva. 
pericardio; el primero fue negativo, y el segundo presentó fibrosis difusa e infiltración linfocitaria. Se consideraron estos hallazgos compatibles con tuberculosis, aun frente a la ausencia de granulomas típicos, por tratarse de un proceso crónico. Se realizó el catastro familiar con radiografía de tórax y $P P D$, y no se hallaron otros casos de tuberculosis.

Por buena evolución clínica y con diagnóstico de pericarditis constrictiva de origen tuberculoso, se decidió su egreso hospitalario y se indicó el tratamiento con drogas tuberculostáticas por 12 meses, furosemida $(20 \mathrm{mg})$, carvedilol (3,25 mg) y enalapril (2,5 mg).

Actualmente, se encuentra asintomático y realiza los controles anuales en el Servicio de Cardiología Infantil.

\section{DISCUSIÓN}

La pericarditis constrictiva es una entidad poco frecuente, con una prevalencia del $1 \%$ en los adultos y desconocida en los niños, ${ }^{4}$ razón por la cual no suele considerarse dentro de los diagnósticos diferenciales iniciales.

FIgURA 2. Resección quirúrgica

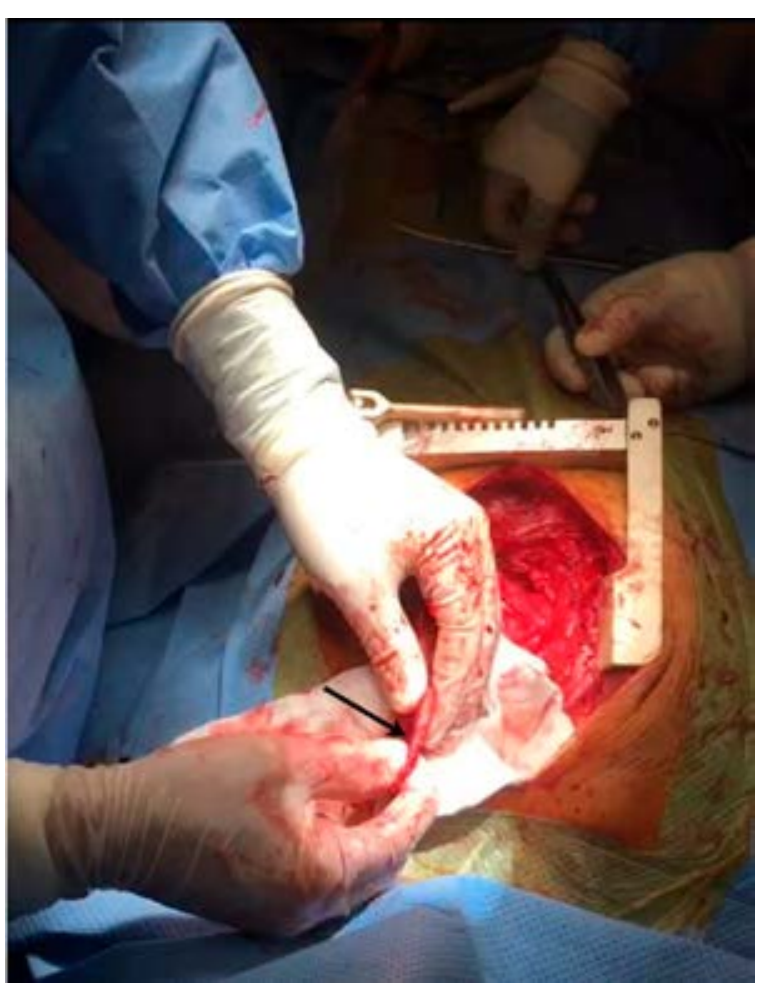

En la foto, se puede observar el grosor aumentado del pericardio resecado.
Se trata de la presencia de un pericardio rígido que impide la correcta función diastólica, lo que lleva a una insuficiencia cardíaca congestiva progresiva, que suele manifestarse con ascitis, derrame pleural, ingurgitación yugular y, en raras ocasiones, hepatomegalia congestiva. Por su forma de presentación, generalmente, simula otras patologías, como cirrosis hepática, miocardiopatía restrictiva o insuficiencia cardíaca. $^{5}$

Sus causas son múltiples: se encuentra, en primer lugar, la pericarditis constrictiva idiopática, en la que no se logra hallar un agente etiológico claro, ${ }^{4,6}$ seguida por la tuberculosis, que constituye la primera causa de origen infeccioso, sobre todo, en los países no desarrollados. En estos casos, el compromiso pericárdico se debe a la extensión directa de la infección desde los ganglios linfáticos mediastinales o el miocardio, o por diseminación hematógena. ${ }^{6}$ Puede también ser secundaria a una pericarditis aguda viral y presentarse incluso varios años después de que se resuelve. ${ }^{7}$ El antecedente de radioterapia o cirugía cardiovascular también debe ser tenido en cuenta como causa probable. ${ }^{8}$

Los pacientes suelen encontrarse asintomáticos u oligosintomáticos, y el principal síntoma es la disnea. Por ello, a pesar del avance en los métodos de diagnóstico, es frecuente observar un retraso diagnóstico de hasta 24 meses desde el inicio de los síntomas. ${ }^{5}$

Ante la duda diagnóstica, es necesario implementar métodos complementarios que confirmen la sospecha. El análisis de laboratorio suele ser normal, aunque puede hallarse anemia, elevación de reactantes de fase aguda y un patrón colestásico en el hepatograma debido a la congestión hepática. En el electrocardiograma, por lo general, se encuentran cambios no específicos en la onda T y un patrón de bajo voltaje. ${ }^{8} \mathrm{El}$ signo ecocardiográfico más específico es la disquinesia del tabique interventricular, y se observa también engrosamiento pericárdico, dilatación y ausencia de colapso de la VCI en inspiración, variaciones respiratorias del flujo de la válvula mitral (> 25\%) y tricuspídea (> $40 \%$ ) y dilatación biauricular. ${ }^{9}$ Los estudios por imágenes, como la resonancia magnética cardíaca y la tomografía computada, son la clave del diagnóstico. ${ }^{10}$ En ellos, puede evidenciarse engrosamiento pericárdico (es patológico un espesor mayor de $2 \mathrm{~mm}$ ) y calcificaciones pericárdicas. ${ }^{1,6}$ Esto último puede observarse también, aunque de manera infrecuente, en la radiografía de tórax. ${ }^{10} \mathrm{En}$ 
estudios realizados con contraste, puede haber dilatación de la VCI y las venas suprahepáticas. ${ }^{1,8}$ El cateterismo cardíaco no es un estudio utilizado de rutina; es necesario únicamente frente a dudas diagnósticas. ${ }^{6,10}$ Para el diagnóstico etiológico, es imprescindible la biopsia pericárdica con búsqueda de Micobacterium tuberculosis por métodos directos y cultivo o la detección de ácido desoxirribonucleico del bacilo en el líquido pericárdico. En los países donde la tuberculosis es prevalente, puede implementarse el tratamiento tuberculostático empírico frente a casos sospechosos ${ }^{6}$ en adición a la cirugía.

El tratamiento de primera línea es la pericardiectomía, ${ }^{11}$ que, a pesar de ser una cirugía de alto riesgo y gran dificultad técnica, resulta curativa en la gran mayoría de los pacientes. Es fundamental realizarla en forma temprana para evitar complicaciones miocárdicas irreversibles. ${ }^{12}$ Son indicadores de peor pronóstico la presencia de signos de enfermedad avanzada antes de la cirugía, tales como calcificaciones pericárdicas, hepatomegalia, falla renal, hipertensión pulmonar o alteraciones en la función miocárdica. Se ha evidenciado también como factor de mal pronóstico la demora de 6 meses o más desde el inicio de los síntomas hasta la cirugía. La sobrevida suele ser excelente para aquellos con pericarditis constrictiva idiopática o de etiología infecciosa.

\section{REFERENCIAS}

1. Marshal A, Ring N, Lewis T. Constrictive pericarditis: lessons from the past five years' experience in the South West Cardiothoracic Centre. Clin Med (Lond). 2006; 6(6):592-7.

2. Talwar S, Nair VV, Choudhary SC, Screeniwas V, et al. Pericardiectomy in children $<15$ years of age. Cardiol Young. 2014; 24(4):616-22.

3. Chen MR, Wu SJ, Yun CH. Constrictive Pericarditis. Pediatr Cardiol. 2009; 30(7):1032-3.

4. Backhoff D, Steinmetz M, Ruschewski W, Stastny B, et al. Severe Constrictive Pericarditis After Parvovirus B19 and Human Herpes Virus 6 Infection in a 9-Year-Old Girl. Pediatr Cardiol. 2013; 34(8):2089-92.

5. Fernandes F, Melo DT, Ramires FJ, Dias RR, et al. Importance of Clinical and Laboratory Findings in the Diagnosis and Surgical Prognosis of Patients with Constrictive Pericarditis. Arq Bras Cardiol. 2017; 109(5):457-65.

6. Ak K, Demirbaş E, Ataş H, Birkan Y, et al. Results of pericardiectomy for constrictive pericarditis: single- center experience. Herz. 2017; 42(1):75-83.

7. Kytö V, Sipilä J, Rautava P. Chronic constrictive pericarditis in general adult population. Int J Cardiol. 2014; 176(3):1158-60.

8. Mittal SR. Occult Constrictive Pericarditis. J Assoc Physicians India. 2014; 62(3):279-81.

9. Chen MY, Chen YJ, Chen SJ, Lu CW. A Girl With Right Heart Related Failure Related to Effusive-Constrictive Pericarditis. Pediatr Cardiol. 2011; 32(5):681-4.

10. Porta-Sánchez A, Sagristá-Sauleda J, Ferreira-González I, Torrents-Fernández A, etal.ConstrictivePericarditis:Etiologic Spectrum, Patterns of Clinical Presentation, PrognosticFactors, and Long-term Follow-up. Rev Esp Cardiol (Engl Ed). 2015; 68(12):1092-100.

11. Thompson JL, Burkhart HM, Dearani JA, Cetta F, et al. Pericardiectomy for Pericarditis in the Pediatric Population. Ann Torac Surg. 2009; 88(5):1546-50.

12. Zhu P, Mai M, Wu R, Lu C, et al. Pericardiectomy for constrictive pericarditis: single-center experience in China. J Cardiothorac Surg. 2015; 10:34. 